\title{
High expression of PD-L1 Predicts Worse Overall Survival in the Cavitary Lung Adenocarcinoma
}

Jiangyong Liu ( $\sim$ liuyong4327939@163.com )

General Hospital of central Theater Command

Mingming Gu

General Hospital of Central Theater Command

Yang Xue

General Hospital of Central Theater Command

Yong Ren

General Hospital of Central Theater Command

Wencai Huang

General Hospital of Central Theater Command

\section{Research}

Keywords: Lung adenocarcinoma, cavity, programmed cell death ligand-1, tumor infiltrating lymphocytes, multiplex immunohistochemistry

Posted Date: December 21st, 2020

DOl: https://doi.org/10.21203/rs.3.rs-131097/v1

License: (c) (i) This work is licensed under a Creative Commons Attribution 4.0 International License.

Read Full License 


\section{Abstract \\ Objective}

Solitary cavitary lung cancer is one of the rare types of lung cancer. Generally, the relationship between cavitary lung adenocarcinoma and immunotherapy remains unknown. We aimed to assess programmed cell death ligand-1(PD-L1) expression and CD8-positive (CD8 ${ }^{+}$) tumor infiltrating lymphocytes (TILs) density, and evaluate their prognostic significance of patients with cavitary lung adenocarcinoma (LUAD).

\section{Methods}

65 patients diagnosed as solitary cavitary LUAD were included in this study, 30 cases of noncavitary LUAD patients were collected as controls, and their specimens from surgery or biopsy were obtained. Expression of PD-L1 protein and CD8 $8^{+}$TILs were detected by traditional immunohistochemistry and multiplex quantitative immunofluorescence technology. The correlations of PD-L1 expression and clinicopathological features, including overall survival in cavitary LUAD patients was evaluated based on the follow-up data.

\section{Results}

Overexpression of PD-L1 protein was detected in the tumor tissues of cavitary LUAD patients compared to the noncavitary LUAD controls. PD-L1 expression level was significantly related to the lymph node $(P=$ $0.001)$, TNM stage $(P=0.024)$, and $C D 8^{+}$TIL status $\left(r_{s}=-0.272, P=0.025\right)$. High PD-L1 expression predicted high mortality rate $(P<0.001)$, but $C D 8^{+}$TIL group showed better survival in cavitary LUAD patients $(P=0.011)$. This phenotype with high PD-L1 expression and low CD8 + TIL predicted poorer overall survival of the patients with cavitary LUAD, compared to the other phenotypes. Moreover, $\mathrm{CD} 8^{+} \mathrm{TIL}$ was an independent good prognosis factor.

\section{Conclusion}

We firstly demonstrated that PD-L1 is upregulated in the cavitary LUAD patients, and high expression of PD-L1 negatively correlated with CD8 T cell infiltrating status. High PD-L1 expression and low CD8 + TIL can predict poorer overall survival of the patients with cavitary LUAD.

\section{Introduction}

In both sexes combined, lung cancer is the most common type of cancer diagnosed and the leading cause of cancer death worldwide in 185 countries[1]. Among all types of lung cancer, cavitary lung cancer is particularly unique and seldom reported [2], which occurs in $8 \%$ of all lung cancers [3], while other 
researchers reported the incidence rate of $1.00-2.07 \%$ [2]. Cavitation in a tumor nodule is previously thought to be more prevalent in patients with lung squamous cell carcinoma [4]. Following with LUAD increases, cavitary LUAD has also been reported, with an incidence of 5.7 to $14.9 \%$ in patients with LUAD [5]. As a rare type of lung cancer, cavitary lung cancer is not easily diagnosed by radiological measures, and has a worse prognosis because of high TNM stage, compared with noncavitary lung cancer [6-9]. Moreover, the biological features of the underlying walled cavity are still poorly understood.

Immunotherapy is regarded as a novel choice in the treatment of a variety of cancers with poor prognosis [10]. The development of immune checkpoint inhibitors has changed the treatment of non-small cell lung cancer (NSCLC) [11]. As one of the typical checkpoint inhibitors, programmed cell death 1 (PD-1) is an inhibitory cell-surface receptor that is expressed on activated T-cells and other immune cells. Anticancer immunotherapy targeting immune checkpoints with antibodies to PD-1 and its ligand PD-L1 is an established treatment modality for NSCLC $[12,13]$. One of the important mechanisms that anti-PD-L1 monoclonal antibodies restraint the lymphocyte inhibition by binding to the PD-1 receptor, which preventing the PD-1 binding with its ligands 1 or 2 (PD-L1 or PD-L2), and permit T cells to maintain their tumor cell killing function $[14,15]$. PD-L1-positive patients had a higher chance of achieving an objective response when treated with anti-PD-L1 monoclonal antibodies $[16,17]$.

Tumor infiltrating lymphocytes (TILs) also play a vital role in predicting tumor progression in different kinds of cancers [18]. As the most studied component of tumor-associated immune response, the cytotoxic CD8-positive (CD8+) T cells expression could predict better prognosis in patients with breast or ovarian cancers $[19,20]$. However, the relationship between solitary thin-walled cavity lung cancer and immune checkpoint remains unknown. Therefore, in this study, we take advantage of human specimens to assess PD-L1 expression and CD8 + TIL density, and investigate its prognostic significance in cavitary LUAD.

\section{Material And Methods}

\section{Samples collection}

65 patients who were diagnosed as the cavitary lung cancer in the period from September 2005 and October 2015 in the General Hospital of Central Theater Command Hospital, PLA were included in the research, and they had no neoadjuvant therapy before surgical resection. All patients underwent the 64row spiral computed tomography with slice thickness of $1.25 \mathrm{~mm}, 1.5 \mathrm{~mm}$ or HRCT. Two radiologists (J Liu and $Y$ Xue) examined the imaging features of these samples independently. According to previous studies, the tumor cavitation was defined as an air-filled space with a shortest diameter of $\geq 5 \mathrm{~mm}$ within a tumor $[5,21,22]$ (Fig. 1).

Formalin-fixed, paraffin-embedded (FFPE) tissues of 65 patients with the cavitary LUAD were collected. Two pathologists ( $Q$ Wang and $Y$ Ren) reconfirmed the histopathologic features of each sample 
independently. Another 30 cases of general LUAD patients who didn't present as a solitary cavity and excluded post-neoadjuvant therapy were collected as controls.

Clinicopathological data were retrieved from clinical records and histopathology reports. The follow-up began on the date of surgery or biopsy and ended in October 2018. The median follow-up was 45 months (1-115 months). Overall survival was defined as the period from diagnosis to death or the end of followup. LUAD specimens were classified according to the 8th edition of TNM classification by Union for International Cancer Control/ American Joint Committee on Cancer (2017)[23].

Immunohistochemical analysis.

After deparaffinized and rehydrated, antigen retrieval was applied in citrate $(10 \mathrm{mM}, \mathrm{pH} 6.0)$ at $95^{\circ} \mathrm{C}$ for 15 minutes by microwave. PD-L1 and CD8 expression in FFPE tumor sections was performed by IHC using primary rabbit anti-human PD-L1 polyclonal antibody (1:100 dilution, E1L3N, Cell Signaling Technology, USA) and mouse anti-human CD8 monoclonal antibody (ready-to-use, C8/144B, Dako, Agilent, USA) at $4{ }^{\circ} \mathrm{C}$ overnight. Then horseradish peroxidase (HRP) conjugated to the goat antimouse/rabbit second antibody (Dako REAL EnVision Detection System, Agilent, USA) was incubated with PD-L1 and CD8 at $37^{\circ} \mathrm{C}$ for 30 minutes. Subsequently, the sections were added with 3,3'-

diaminobenzidine (DAB) chromogen (Dako, Agilent, USA) as the chromogen and nuclear counterstaining with hematoxylin.

\section{Evaluation of immunohistochemistry}

Immunostaining intensity was observed using light microscopy (Olympus BX-53 with CCD DP73). Results were scored by two pathologists ( $Q$ Wang and $Y$ Ren) who were independent and blinded to the clinicopathological characteristics of the research.

The immunohistochemistry characteristics and cut-offs of PD-L1 for being regarded as positive varies in different research. Here, the cut-off of PD-L1 protein expression in tumor cells were defined as 5\%. PD-L1 $\geq 5 \%$ was regarded as high expression, which was consistent with many types of cancers $[24,25]$.

For the CD8 evaluation, CD8 ${ }^{+}$TILs were counted in each slide at $\times 200$ magnification. The mean of the three counts was calculated for each case and the cutoff point of high or low expression was determined on the median number of total scores[20, 26].

\section{Multiplex immunofluorescence staining}

Manual multiplex immunofluorescence (mIF) staining was performed in 4- $\mu \mathrm{m}$ sections obtained from FFPE lung cancer blocks by using the Opal 4-Color IHC Kit (PerkinElmer, Waltham, MA)[11]. The stained slides were scanned by a Vectra multispectral microscope (Akoya Biosciences, USA). The immunofluorescence markers were consisted of PD-L1 (E1L3N, dilution 1:200; Cell Signaling Technology, USA), CK (AE1/AE3) and CD8(C8/144B) are ready-to-use antibodies from Agilent/DAKO, California, USA. 
Primary antibody was visualized by using tyramide signal amplification linked to a specific fluorochrome from the multiplex IHC Kit for each primary antibody. A stripping procedure, based on the Meidi microwave (Meidi, China), was performed for each consecutive antibody staining. Human tonsil FFPE tissues were also used with and without primary antibodies as positive and negative (autofluorescence) controls, respectively. The mIF-stained slides were scanned with a Vectra 2.3 microscope system (Akoya Biosciences, USA) under fluorescent illumination. From each slide, Vectra automatically captured the fluorescent spectra from $420 \mathrm{~nm}$ to $720 \mathrm{~nm}$ at 20-nm intervals with the same exposure time and then combined the captured images to create a single stack image that retained the particulate spectral signature of all IF markers.

\section{Statistical analysis}

Data were expressed as frequencies for categorical variables and mean \pm SD for numerical variables. SPSS 21.0 software (Chicago, IL, USA) was used to perform all statistical analyses. $\chi^{2}$ test or Fisher exact test were carried out to evaluate the correlations of PD-L1 expression and clinicopathological parameters of cavitary LUAD patients. We explored the relationship between PD-L1 and CD8 ${ }^{+}$TILs using Spearman correlation analysis. The survival analysis was assessed using the Kaplan-Meier curve and log-rank test to the statistical difference survival data. Cox proportion hazard regression model was conducted to evaluate univariate and multivariate analysis of survival as well as the independent prognostic values. $P$ values $<0.05$ were considered statistically significant.

\section{Results}

\section{Patient characteristics}

As showed in Table 1, among the 65 cavitary LUAD patients, 36 (55.4\%) were male and 29 (44.6\%) were female, with the mean age of 58 years old (range 48-71). 31 (47.7\%) patients were alive and $34(52.3 \%)$ died at the end of follow-up. The data of T, N, M and TNM stage as well as the clinicopathological parameters of noncavitary LUAD patients also showed in Table 1.

\section{$P D-L 1$ and CD8 protein expression}

As showed in Fig.2-4, PD-L1 and CD8 proteins expressed in both cavitary LUAD tissues and noncavitary LUAD tissues. In tumor tissues, PD-L1 was located on the membrane and the cytoplasm of tumor cells, and the cytoplasm of immune cells (Fig.2A, B and Fig.3 A, B, Fig.4B, D). CD8 positive T cells were observed inside and outside tumor nest of LUAD (Fig.2C, D and Fig.3 C, D, Fig.4C, F).

In 65 cases of cavitary LUAD tissues studied, 55.4\% ( $n=36)$ cases showed low PDL1 expression, and $44.6 \%(n=29)$ cases of high PD-L1 expression was found. While in 30 cases of noncavitary LUAD tissues, $80 \%(n=24)$ were PD-L1 low expression and 20\% $(n=6)$ were PD-L1 high expression. $\chi 2$ test showed positive rates of PD-L1 in the cavitary LUAD tissues was significantly higher than that of noncavitary LUAD tissues $(P=0.021$, Table 2$)$. 
Among 65 cases of cavitary LUAD patients, 48(73.9\%) cases were CD8 negative and 17(26.1\%) were CD8 positive. In 30 cases of noncavitary LUAD tissues, 12(40.0\%) were CD8 negative and 18(60.0\%) were CD8 positive. $\chi 2$ test showed the CD8+ TILs in the cavitary LUAD tissues were significantly reduced, compared to the noncavitary controls $(P=0.022$, Table 2$)$.

\section{PD-L1 expression and clinicopathological parameters in cavitary LUAD patients}

The relationship between tumor PD-L1 expression and clinicopathologic variables in the cavitary LUAD was investigated by $\chi^{2}$ test. As listed in Table 3, high expression of PD-L1 protein was significant correlated with the lymph node metastasis $(N)(P=0.001)$ and TNM stage $(P=0.024)$. Negative correlation was observed between PD-L1 and CD8 TIL status ( $r s=-0.272, P=0.025)$. However, PD-L1 expression was not significantly associated with age, gender, tumor size in the cavitary LUAD patients.

\section{Prognostic value of PD-L1 expression in cavitary LUAD patients}

Survival analysis determined by Kaplan-Meier curve and log-rank test was determined to investigate the prognostic value of PD-L1 expression and CD8 TIL status in cavitary LUAD patients. High expression of PD-L1 group predicted poorer survival and high mortality rate in cavitary LUAD patients (Fig.5A, $P=0.004$ ). CD8+ TIL group showed better survival in cavitary LUAD patients (Fig.5B, $P=0.001$ ). Moreover, patients with high PD-L1 expression and low CD8+ TIL demonstrated poorer overall survival than that with the other phenotypes (Fig.5C, $P=0.001$ ).

In univariate analysis, both PD-L1 and CD8 expression levels were found to be significantly related to the overall survival (OS) of LUAD patients (HR and 95\% $\mathrm{Cl}=2.670,1.334-5.345, \mathrm{P}=0.006 ; \mathrm{HR}$ and $95 \%$ $\mathrm{Cl}=0.995,0.991-0.998, \mathrm{P}=0.002$, respectively. Table 4). Moreover, the phenotype of high PD-L1 expression and low CD8+ TIL had higher risk than the other phenotype (HR and 95\% $\mathrm{Cl}=2.999,1.518-5.923$, $\mathrm{P}=0.002$ ). Simultaneously, TNM stage was significantly correlated with the OS of LUAD (Table 4). In order to analyze whether the above univariate was an independent prognostic factor, a multivariate COX proportional hazard model on OS was performed. The clinicopathological characteristics and the two protein expressions were added in the multivariate analysis model. The results indicated that only CD8 expression was an independent prognosis parameter in OS of LUAD patients ( $\mathrm{HR}$ and $95 \% \mathrm{Cl}=0.005$, $0.007-0.401, P=0.004$, Table 4).

\section{Discussion}

To our knowledge, the current study was the first to investigate that high expression of PD-L1 protein in the cavitary LUAD tissues, compared to the noncavitary LUAD tissues. PD-L1 expression level was significantly correlated to lymph node metastasis, TNM stage and CD8 TIL status. Interestingly, our study showed that high PD-L1 expression and low CD8 + TIL can predict poorer overall survival of the patients with cavitary LUAD. CD8 + TIL had an independent predictor of LUAD prognosis. 
The examinations of chest radiography and CT are common used for the clinical diagnosis of lung cancer. Radiographic features of cavities that suggest malignancy include multiple holes, a nodular illdefined inner or outer wall, and an eccentric excavation with irregular margins[27]. Cavitary lung cancers are more prevalent in patients with worse survival than that for noncavitary NSCLC patients [5, 22], because of advanced tumor stage, and vascular, lymphatic, or pleural invasion of cavitary LUAD [5]. Currently, there is a lack of cognition concerning its onset and progression for solitary cavitary LUAD, thus, this rare type of cancer is subject to misdiagnosis and missed diagnosis [2]. Necrosis may cause solitary cavity because of primary cancer overgrowth. The lesion may originate from the distal part of lung or a preexisting cystic lesion. Tumor growth leads to bronchial obstruction and vascular invasion, which provides an environment of ischemia and hypoxia, and then resulting in the tumor necrosis. Furthermore, the autophagy of neoplastic cell can also induce the cavitary lesion $[6,9]$.

Immune checkpoint inhibitors targeting the PD-1/PD-L1axis have shown promising results in patients with NSCLC. Overexpression of PD-L1 is associated with poor recurrence-free survival and overall survival [28]. Pembrolizumab has been approved as first-line treatment for advanced PD-L1 positive NSCLC patients [29]. In our study, we found that high expression of PD-L1 was detected in 44.6\% (29/65) cases of cavitary LUAD, significantly increased, compared to $20 \%(6 / 30)$ cases of noncavitary lung cancer, which demonstrated tumor necrosis correlates with higher PD-L1 expression in the LUAD[30]. Moreover, high expression of PD-L1 was correlated with high TNM stage, and could predict poor prognosis of patients with cavitary LUAD. The above results confirmed that high expression of PD-L1 may promote malignant progression, and regard as one of cancer immunotherapy targets of the cavitary LUAD. However, recent paper have reported high expression of PD-L1 is also positively asscioated with mutations in KRAS, TP53, and MET, however, negatively associated with mutations of EGFR and STK11 of LUAD[31]. Thus, we had to further the specific molecular features of cavitary LUAD to analyze the mechanism of malignant progression and poor prognosis.

Inflammation is one of the notable features of cancer, and can lead to tumor progression [32]. Cytokines also has an anti-tumor immune effect, IFN- $a$, IFN- $y$, and TNF- $a$ can increase expression of PD-L1 in a variety of cancers [33]. In addition, $\mathrm{CD} 8^{+} \mathrm{T}$ cells also correlate with PD-L1 expression and participated in the inflammation of anti-tumor immune response [34,35]. Our results also confirmed that negative correlation was observed between high expression of PD-L1 and CD8 + TIL in cavitary LUAD. Moreover, this phenotype with high PD-L1 expression and low CD8 + TIL could predict poorer overall survival of the patients with cavitary LUAD, compared to the other phenotypes (such as low PD-L1 and high CD8 + TIL). Moreover, CD8 + TIL was an independent marker to predict prognosis of LUAD. In a comprehensive view, it suggested that inflammation or necrosis induced by the dysregulation of tumor growth, and then activates the cytokines secretion as well as leads to the formation of thin-walled cavity lesion in the LUAD development. Subsequently, cytokines increase the expressions and activities of PD-L1, which contributes tumor cells escaping from immune surveillance, further promote malignant progression of cavitary LUAD. $\mathrm{CD} 8^{+}$TILs involved in inflammation and immune processes described above. Further studies are required 
to elucidate the mechanisms of PD-L1 overexpression and its relationship with inflammation or necrosis in solitary cavity LUAD cases.

In conclusion, we firstly demonstrated that PD-L1 expression is upregulated in the cavitary LUAD patients, and high expression of PD-L1 negatively correlates with CD8 T cell infiltrating status. High PD-L1 expression and low CD8 + TIL can predict poorer overall survival of the patients with cavitary LUAD. Our results illustrate that PD-L1 is a critical immune checkpoint and improve our mechanistic understanding of cavitary LUAD.

\section{Declarations}

\section{Authors' contributions}

JYL and WCH initiated and designed the work, supervised the data collection. JYL and MMG prepared the manuscript and performed the experiments. $Y X$ and $Y R$ contributed to the acquisition of patients and tissues specimens and to the analysis and interpretation of data. All authors read and approved the final manuscript.

\section{Funding}

This work was supported by grants from the Natural Science Foundation of Hubei Province General Project (grant number: 2020BCB059).

\section{Availability of data and materials}

The data sets and/or analyzed during the current study are available from the corresponding author on reasonable request.

\section{Ethics approval and consent to participate}

This research was approved by the Ethics Committee of General Hospital of Central Theater Command, PLA, Wuhan (2018-002-1). All patient specimens and clinical data involved in this study complied with the Declaration of Helsinki.

\section{Consent for publication}

Written informed consent was obtained from a legally authorized representative(s) for anonymized patient information to be published in this article.

\section{Competing interests}

The authors state that there are no conflicts of interest to disclose.

\section{Acknowledgements}


Not applicable.

\section{References}

1. Bray F, Ferlay J, Soerjomataram I, Siegel RL, Torre LA, Jemal A: Global cancer statistics 2018: GLOBOCAN estimates of incidence and mortality worldwide for 36 cancers in 185 countries. $C A$ Cancer J Clin 2018, 68(6):394-424.

2. Qi Y, Zhang Q, Huang Y, Wang D: Manifestations and pathological features of solitary thin-walled cavity lung cancer observed by CT and PET/CT imaging. Oncol Lett 2014, 8(1):285-290.

3. Woodring JH, Fried AM, Chuang VP: Solitary cavities of the lung: diagnostic implications of cavity wall thickness. AJR Am J Roentgeno/ 1980, 135(6):1269-1271.

4. Gasinska A, Kolodziejski L, Niemiec J, Dyczek S: Clinical significance of biological differences between cavitated and solid form of squamous cell lung cancer. Lung Cancer 2005, 49(2):171-179.

5. Watanabe Y, Kusumoto M, Yoshida A, Shiraishi K, Suzuki K, Watanabe SI, Tsuta K: Cavity Wall Thickness in Solitary Cavitary Lung Adenocarcinomas Is a Prognostic Indicator. Ann Thorac Surg 2016, 102(6):1863-1871.

6. Chaudhuri MR: Primary pulmonary cavitating carcinomas. Thorax 1973, 28(3):354-366.

7. Onn A, Choe DH, Herbst RS, Correa AM, Munden RF, Truong MT, Vaporciyan AA, Isobe T, Gilcrease MZ, Marom EM: Tumor cavitation in stage I non-small cell lung cancer: epidermal growth factor receptor expression and prediction of poor outcome. Radiology 2005, 237(1):342-347.

8. Coffey JP, Hill JC: 18F-fluoro-2-deoxy-D-glucose standardized uptake value in cavitating non-smallcell lung carcinoma. Nucl Med Commun 2008, 29(12):1040-1045.

9. Farooqi AO, Cham M, Zhang L, Beasley MB, Austin JH, Miller A, Zulueta JJ, Roberts H, Enser C, Kao SJ et al: Lung cancer associated with cystic airspaces. AJR Am J Roentgenol 2012, 199(4):781-786.

10. Iwai Y, Ishida M, Tanaka Y, Okazaki T, Honjo T, Minato N: Involvement of PD-L1 on tumor cells in the escape from host immune system and tumor immunotherapy by PD-L1 blockade. Proc Natl Acad Sci U S A 2002, 99(19):12293-12297.

11. Long L, Zhao C, Ozarina M, Zhao X, Yang J, Chen H: Targeting Immune Checkpoints in Lung Cancer: Current Landscape and Future Prospects. Clin Drug Investig 2019, 39(4):341-353.

12. Gettinger S, Horn L, Jackman D, Spigel D, Antonia S, Hellmann M, Powderly J, Heist R, Sequist LV, Smith DC et al: Five-Year Follow-Up of Nivolumab in Previously Treated Advanced Non-Small-Cell Lung Cancer: Results From the CA209-003 Study. J Clin Oncol 2018, 36(17):1675-1684.

13. Reck M, Rodriguez-Abreu D, Robinson AG, Hui R, Csoszi T, Fulop A, Gottfried M, Peled N, Tafreshi A, Cuffe $S$ et al: Pembrolizumab versus Chemotherapy for PD-L1-Positive Non-Small-Cell Lung Cancer. N Engl J Med 2016, 375(19):1823-1833.

14. Ohaegbulam KC, Assal A, Lazar-Molnar E, Yao Y, Zang X: Human cancer immunotherapy with antibodies to the PD-1 and PD-L1 pathway. Trends Mol Med 2015, 21(1):24-33. 
15. Chen DS, Irving BA, Hodi FS: Molecular pathways: next-generation immunotherapy-inhibiting programmed death-ligand 1 and programmed death-1. Clin Cancer Res 2012, 18(24):6580-6587.

16. Abdel-Rahman O: Correlation between PD-L1 expression and outcome of NSCLC patients treated with anti-PD-1/PD-L1 agents: A meta-analysis. Crit Rev Oncol Hematol 2016, 101:75-85.

17. Aguiar PN, Jr., De Mello RA, Hall P, Tadokoro H, Lima Lopes G: PD-L1 expression as a predictive biomarker in advanced non-small-cell lung cancer: updated survival data. Immunotherapy 2017, 9(6):499-506.

18. Fu Q, Chen N, Ge C, Li R, Li Z, Zeng B, Li C, Wang Y, Xue Y, Song X et al: Prognostic value of tumorinfiltrating lymphocytes in melanoma: a systematic review and meta-analysis. Oncoimmunology 2019, 8(7):1593806.

19. Li J, Wang J, Chen R, Bai Y, Lu X: The prognostic value of tumor-infiltrating T lymphocytes in ovarian cancer. Oncotarget 2017, 8(9):15621-15631.

20. Al-Saleh K, Abd El-Aziz N, Ali A, Abozeed W, Abd El-Warith A, Ibraheem A, Ansari J, Al-Rikabi A, Husain S, Nabholtz JM: Predictive and prognostic significance of CD8(+) tumor-infiltrating lymphocytes in patients with luminal B/HER 2 negative breast cancer treated with neoadjuvant chemotherapy. Oncol Lett 2017, 14(1):337-344.

21. Xue X, Wang P, Xue Q, Wang N, Zhang L, Sun J, Wang K, Yang B, Wang J: Comparative study of solitary thin-walled cavity lung cancer with computed tomography and pathological findings. Lung Cancer 2012, 78(1):45-50.

22. Shigefuku S, Kudo Y, Yunaiyama D, Matsubayashi J, Park J, Nagao T, Shimada Y, Saji H, Hagiwara $\mathrm{M}$, Okano $\mathrm{T}$ et al: Prognostic factors for surgically resected non-small cell lung cancer with cavity formation. J Thorac Dis 2018, 10(2):973-983.

23. Detterbeck FC, Boffa DJ, Kim AW, Tanoue LT: The Eighth Edition Lung Cancer Stage Classification. Chest 2017, 151(1):193-203.

24. Herbst RS, Soria JC, Kowanetz M, Fine GD, Hamid O, Gordon MS, Sosman JA, McDermott DF, Powderly JD, Gettinger SN et al: Predictive correlates of response to the anti-PD-L1 antibody MPDL3280A in cancer patients. Nature 2014, 515(7528):563-567.

25. Koganemaru S, Inoshita N, Miura Y, Miyama Y, Fukui Y, Ozaki Y, Tomizawa K, Hanaoka Y, Toda S, Suyama $\mathrm{K}$ et al: Prognostic value of programmed death-ligand 1 expression in patients with stage III colorectal cancer. Cancer Sci 2017, 108(5):853-858.

26. El-Guindy DM, Helal DS, Sabry NM, Abo El-Nasr M: Programmed cell death ligand-1 (PD-L1) expression combined with CD8 tumor infiltrating lymphocytes density in non-small cell lung cancer patients. J Egypt Natl Canc Inst 2018, 30(4):125-131.

27. Xue XY, Liu YX, Wang KF, Zang XF, Sun JP, Zhang MY, Yang B, Ao T, Wang JX: Computed tomography for the diagnosis of solitary thin-walled cavity lung cancer. Clin Respir J 2015, 9(4):392-398.

28. Okita R, Maeda A, Shimizu K, Nojima Y, Saisho S, Nakata M: PD-L1 overexpression is partially regulated by EGFR/HER2 signaling and associated with poor prognosis in patients with non-smallcell lung cancer. Cancer Immunol Immunother 2017, 66(7):865-876. 
29. Meng X, Liu Y, Zhang J, Teng F, Xing L, Yu J: PD-1/PD-L1 checkpoint blockades in non-small cell lung cancer: New development and challenges. Cancer Lett 2017, 405:29-37.

30. Reiniger L, Teglasi V, Pipek O, Rojko L, Glasz T, Vagvolgyi A, Kovalszky I, Gyulai M, Lohinai Z, Raso E et al: Tumor necrosis correlates with PD-L1 and PD-1 expression in lung adenocarcinoma. Acta oncologica 2019, 58(8):1087-1094.

31. Schoenfeld AJ, Rizvi H, Bandlamudi C, Sauter JL, Travis WD, Rekhtman N, Plodkowski AJ, PerezJohnston R, Sawan P, Beras A et al: Clinical and molecular correlates of PD-L1 expression in patients with lung adenocarcinomas. Annals of oncology : official journal of the European Society for Medical Oncology 2020, 31(5):599-608.

32. Dranoff G: Cytokines in cancer pathogenesis and cancer therapy. Nat Rev Cancer 2004, 4(1):11-22.

33. Zhou J, Mahoney KM, Giobbie-Hurder A, Zhao F, Lee S, Liao X, Rodig S, Li J, Wu X, Butterfield LH et al: Soluble PD-L1 as a Biomarker in Malignant Melanoma Treated with Checkpoint Blockade. Cancer Immunol Res 2017, 5(6):480-492.

34. Tumeh PC, Harview CL, Yearley JH, Shintaku IP, Taylor EJ, Robert L, Chmielowski B, Spasic M, Henry G, Ciobanu $V$ et al: PD-1 blockade induces responses by inhibiting adaptive immune resistance. Nature 2014, 515(7528):568-571.

35. Nakazawa N, Yokobori T, Kaira K, Turtoi A, Baatar S, Gombodorj N, Handa T, Tsukagoshi M, Ubukata Y, Kimura A et al: High Stromal TGFBI in Lung Cancer and Intratumoral CD8-Positive T Cells were Associated with Poor Prognosis and Therapeutic Resistance to Immune Checkpoint Inhibitors. Annals of surgical oncology 2020, 27(3):933-942.

\section{Tables}

Table 1. Patient characteristics 


\begin{tabular}{|c|c|c|c|}
\hline Characteristic & Sub-characteristic & Cavitary LUAD(\%) & Noncavitary LUAD (\%) \\
\hline Age & & 58 (range48-71】 & 59.6 (range46-72》 \\
\hline \multirow[t]{2}{*}{ Gender } & Male & $36(55.4)$ & 17(56.7) \\
\hline & Female & $29(44.6)$ & $13(43.3)$ \\
\hline \multirow[t]{2}{*}{ Survival status } & Death & $34(52.3)$ & $12(40.0)$ \\
\hline & Survival & $31(47.7)$ & $18(60.0)$ \\
\hline \multirow[t]{4}{*}{$\mathrm{T}$} & $\mathrm{T} 1$ & $5(7.7)$ & 10(33.3) \\
\hline & $\mathrm{T} 2$ & $50(76.9)$ & 10(33.3) \\
\hline & T3 & $8(12.3)$ & $8(26.7)$ \\
\hline & $\mathrm{T} 4$ & $2(3.1)$ & $2(6.7)$ \\
\hline \multirow[t]{2}{*}{$\mathbf{N}$} & NO & $39(60.9)$ & 19(63.3) \\
\hline & N1-3 & $26(39.1)$ & $11(36.7)$ \\
\hline \multirow[t]{3}{*}{ TNM stage } & I & $28(43.1)$ & $18(60.0)$ \\
\hline & II & $31(47.7)$ & $8(26.7)$ \\
\hline & III & $6(9.2)$ & $4(13.3)$ \\
\hline Total & & 65 & 30 \\
\hline
\end{tabular}

Table 2. PD-L1 expression and CD8 TIL status between cavitary LUAD and noncavitary LUAD.

\begin{tabular}{|c|c|c|c|c|c|c|c|}
\hline & \multicolumn{3}{|c|}{ PD-L1 } & \multirow[t]{2}{*}{$P$} & \multicolumn{2}{|c|}{ CD8 status } & \multirow[t]{2}{*}{$P$} \\
\hline & $\mathrm{n}$ & Low (\%) & High (\%) & & Negative & Positive & \\
\hline & & & & 0.021 & & & 0.022 \\
\hline cavitary lung $A C$ & 65 & $36(55.4)$ & $29(44.6)$ & & $48(73.9)$ & $17(26.1)$ & \\
\hline noncavitary lung $\mathrm{AC}$ & 30 & $24(80.0)$ & $6(20.0)$ & & $15(50.0)$ & $15(50.0)$ & \\
\hline
\end{tabular}

Table 3. Correlations between PD-L1 expression and clinicopathological parameters of patients with cavitary LUAD 


\begin{tabular}{|c|c|c|c|c|}
\hline \multicolumn{2}{|c|}{ Characteristic } & \multicolumn{2}{|l|}{ PD-L1 } & \multirow[t]{2}{*}{$P$ value } \\
\hline & $\mathrm{n}$ & Low (\%) & High (\%) & \\
\hline \multicolumn{5}{|l|}{ Gender } \\
\hline Male & 36 & $19(29.2)$ & $17(26.2)$ & 0.638 \\
\hline Female & 29 & $17(26.2)$ & $12(18.4)$ & \\
\hline \multicolumn{5}{|l|}{ Age } \\
\hline$<=58$ & 31 & 17(26.2) & $14(21.5)$ & 0.933 \\
\hline$>58$ & 34 & 19(29.2) & $15(23.1)$ & \\
\hline \multicolumn{5}{|c|}{ Tumor size } \\
\hline $\mathrm{T} 1+\mathrm{T} 2$ & 55 & $32(49.2)$ & $23(35.4)$ & 0.321 \\
\hline $\mathrm{T} 3+\mathrm{T} 4$ & 10 & $4(6.2)$ & $6(9.2)$ & \\
\hline \multicolumn{5}{|c|}{ Lymph node } \\
\hline NO & 39 & $28(43.1)$ & 11(17.8) & 0.001 \\
\hline N1-3 & 26 & $8(12.4)$ & 18(27.7) & \\
\hline \multicolumn{5}{|c|}{ TNM stage } \\
\hline I & 28 & $20(30.6)$ & $8(12.4)$ & 0.024 \\
\hline II+ III & 37 & $16(24.7)$ & 21(32.3) & \\
\hline CD8 statu & & & & 0.025 \\
\hline Negative & 48 & $23(35.4)$ & $25(38.6)$ & $R s=-0.272$ \\
\hline Positive & 17 & 13(20.0) & $4(6.2)$ & \\
\hline
\end{tabular}

Table 4. COX proportional hazard models on overall survival of LUAD patients. 


\begin{tabular}{|c|c|c|c|c|}
\hline \multirow[t]{2}{*}{ Factors } & \multicolumn{2}{|c|}{ Univariate analysis } & \multicolumn{2}{|c|}{ Multivariate analysis } \\
\hline & $P$ value & $\mathrm{HR} \square 95 \% \mathrm{Cl}[$ & $P$ value & $\mathrm{HR} \square 95 \% \mathrm{Cl} \square$ \\
\hline \multicolumn{5}{|l|}{ Gender } \\
\hline Male vs. Female & 0.769 & & 0.594 & \\
\hline \multicolumn{5}{|l|}{ Age } \\
\hline$<=58$ vs. $>58$ & 0.961 & & 0.253 & \\
\hline \multicolumn{5}{|l|}{ PD-L1 expression } \\
\hline Low vs. High & 0.006 & $2.670(1.334-5.345)$ & 0.055 & \\
\hline \multicolumn{5}{|c|}{ Co-expression of PD-L1 and CD8 } \\
\hline \multicolumn{5}{|c|}{ High PD-L1 and low CD8 vs. the other } \\
\hline & 0.002 & $2.999(1.518-5.923)$ & 0.180 & \\
\hline \multicolumn{5}{|l|}{ CD8 expression } \\
\hline Low vs. High & 0.002 & $0.995(0.991-0.998)$ & 0.004 & $0.055(0.007-0.401)$ \\
\hline \multicolumn{5}{|l|}{ Tumor size(T) } \\
\hline $\mathrm{T} 1, \mathrm{~T} 2$ vs. T3,T4 & 0.449 & & 0.730 & \\
\hline \multicolumn{5}{|c|}{ Lymph node metastasis $(\mathrm{N})$} \\
\hline N0 vs. N1,N2 & 0.127 & & 0.434 & \\
\hline \multicolumn{5}{|l|}{ TNM stage } \\
\hline I,II vs. III & 0.039 & $2.052(1.035-4.068)$ & 0.239 & \\
\hline \multicolumn{5}{|l|}{ Smoking history } \\
\hline Yes vs. No & 0.641 & & 0.755 & \\
\hline
\end{tabular}

\section{Figures}




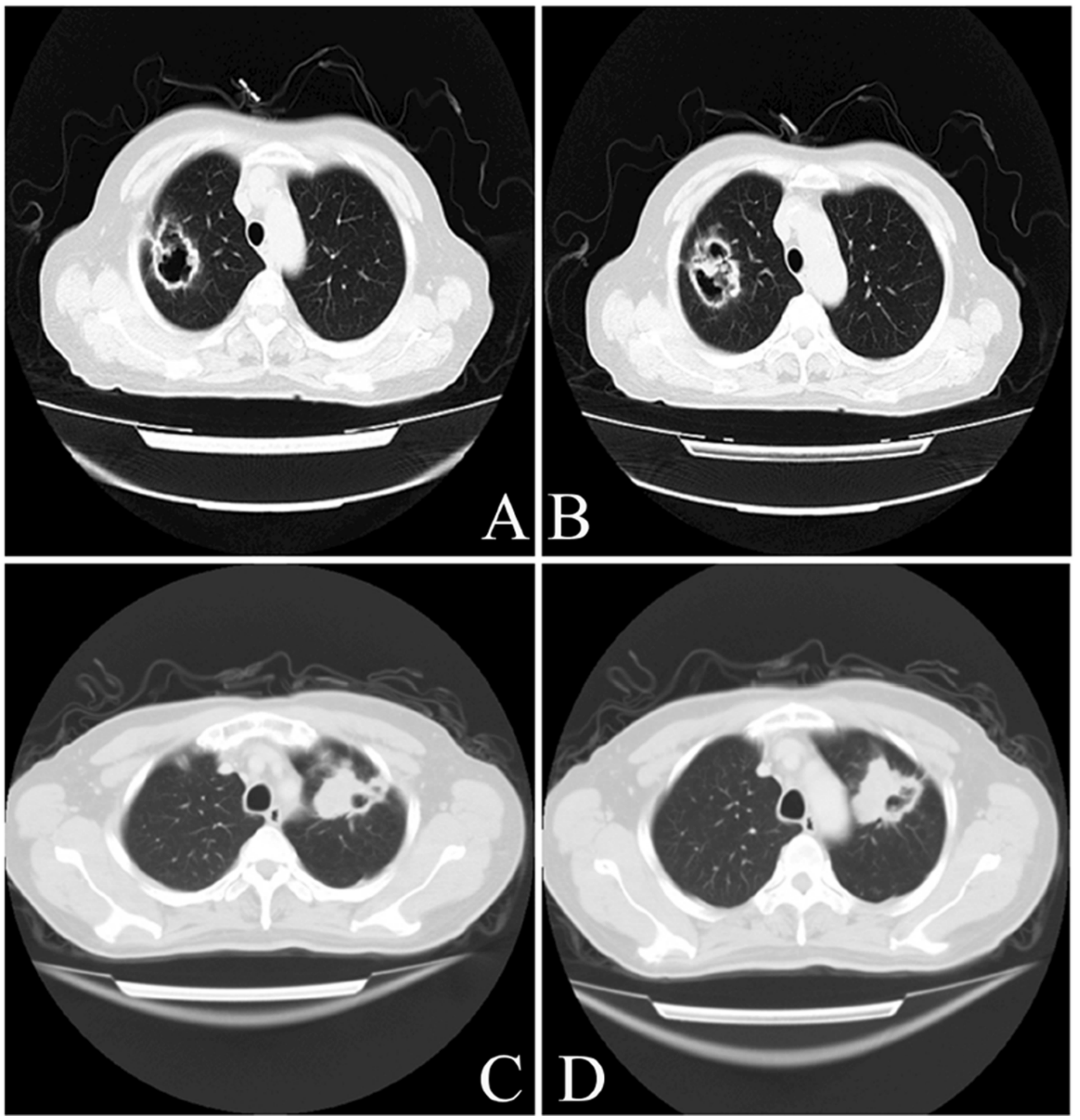

Figure 1

Chest computed tomography (CT) presentation of patients with solitary cavitary lung cancer A-B The posterior segment of the upper lobe of the right lung showed an irregular and shallowly lobulated $40 \times 46$ $\mathrm{mm} 2$ mass, adjacent to pleura traction and depression, with irregular cavity, multiple varying sizes nodules could be seen. C-D The anterior segment of the upper apex of the left lung showed a $46 \times 55 \mathrm{~mm} 2$ soft tissue density shadow, which was lobulated, and pulled by the adjacent pleura, with eccentric cavity. 


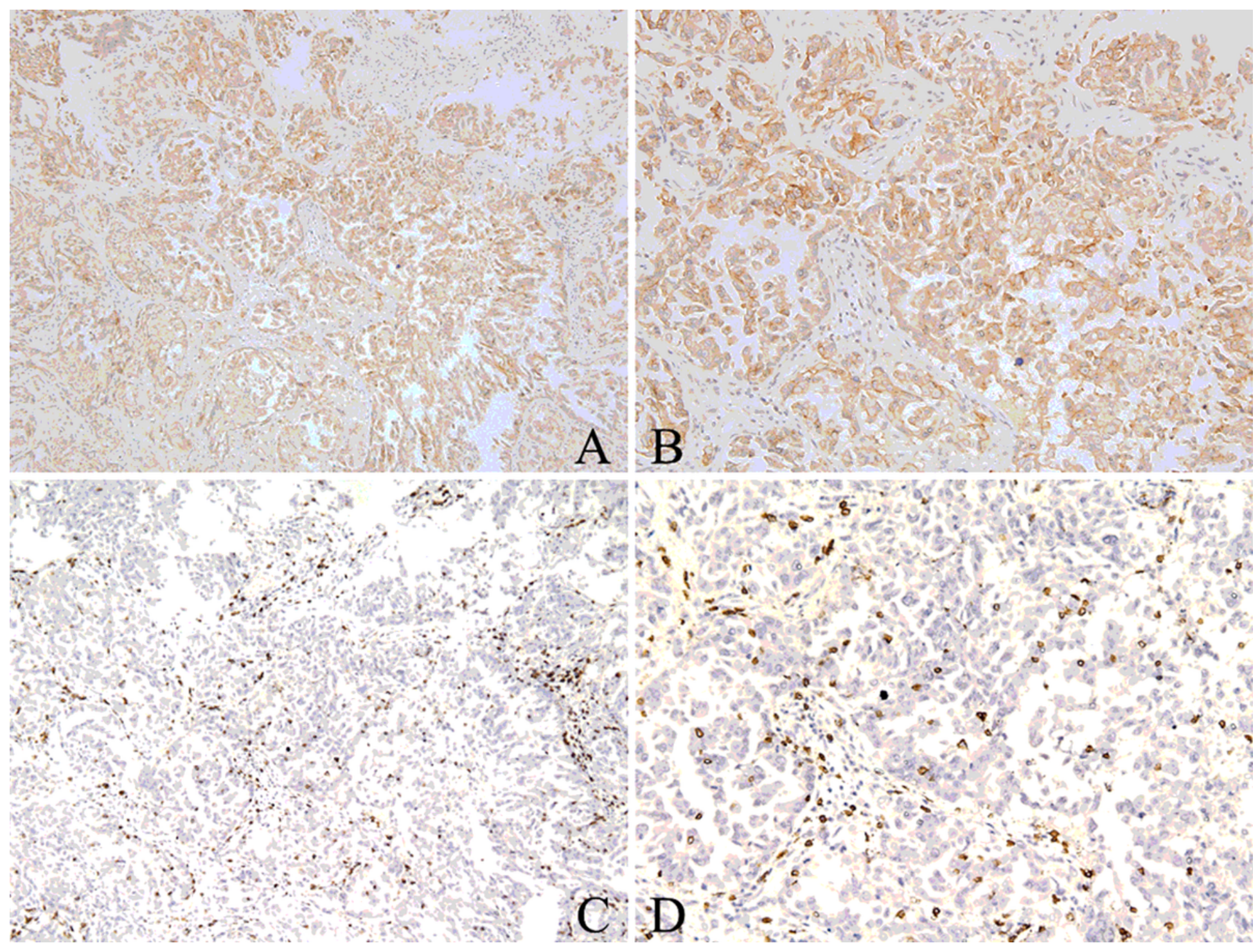

Figure 2

Representative photomicrographs of PD-L1 expression and CD8 T cell infiltration in the cavitary LUAD A and B: high expression of PD-L1 in the tumor cells; $C$ and D: positive expression of CD8 in stroma and tumor islets (Original magnification $A, C \times 100 ; B, D \times 200$ ). 


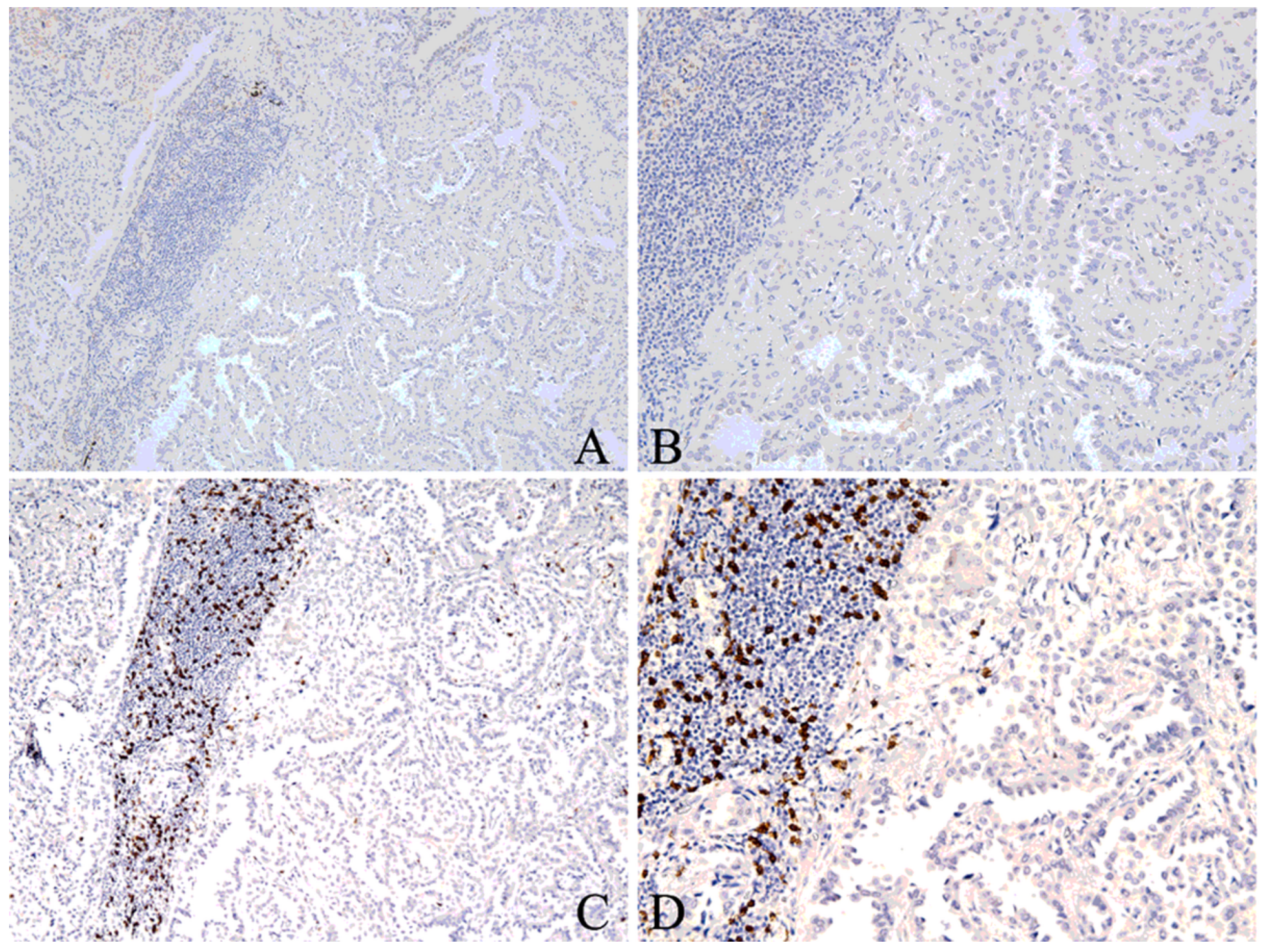

Figure 3

Representative photomicrographs of PD-L1 expression and CD8 T cell infiltration in the noncavitary LUAD $A$ and $B$ : low expression of PD-L1 in the tumor cells; $C$ and D: positive expression of CD8 in stroma and tumor islets, especially in the stroma (Original magnification A, C ×100; B, D ×200). 

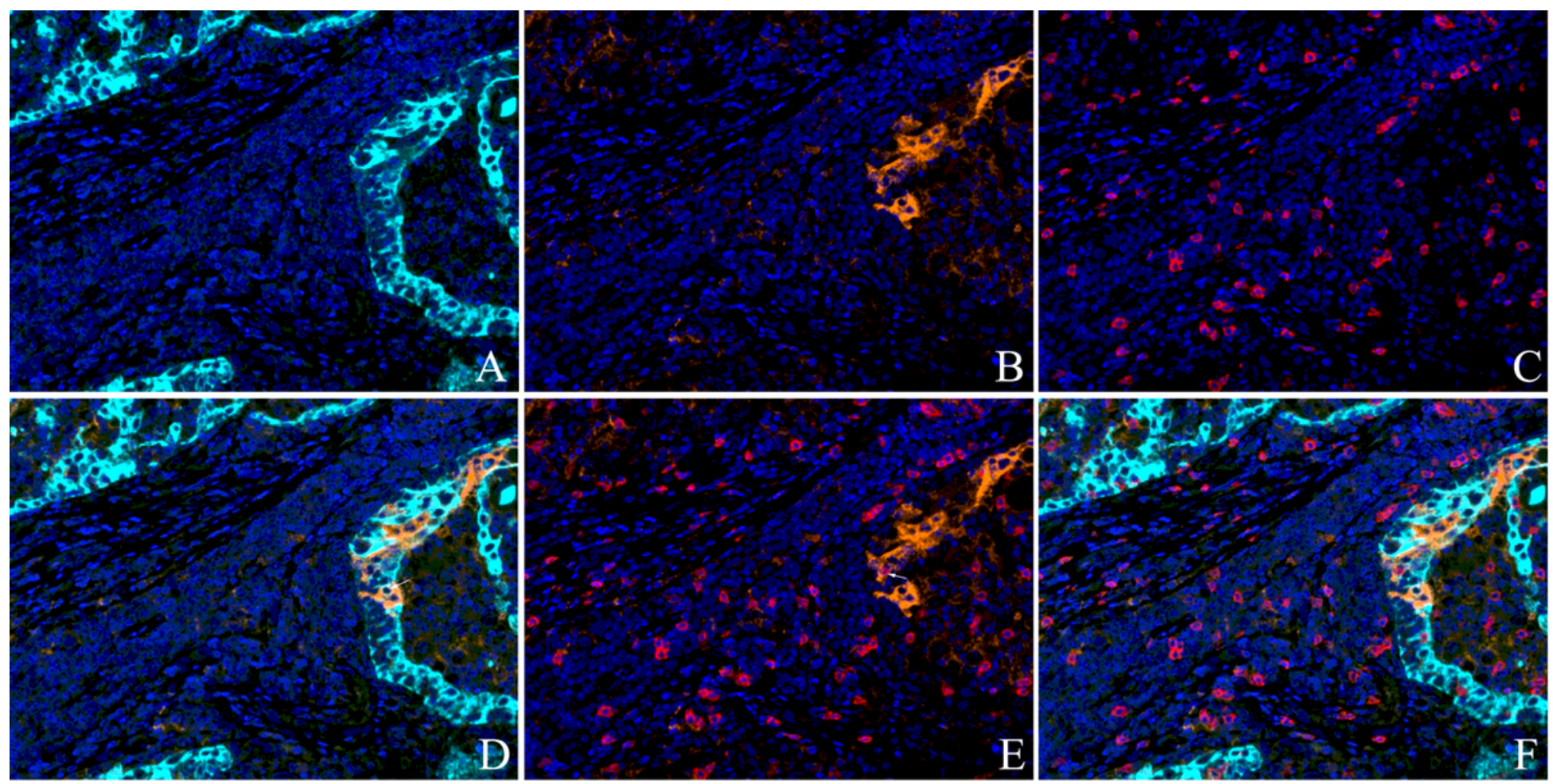

Figure 4

Co-expression of PD-L1 and CD8, CK detected by multiplex immunofluorescence in the cavitary LUAD. A: CK(cyan); B: PD-L1(orange); C: CD8 (red); D: co-expression of PD-L1 and CK (white arrow showed); E: coexpression of PD-L1 and CD3 (white arrow showed); F: unmixed composite image for CK, PD-L1 and CD8 (original magnification of all images $\times 400$ ).
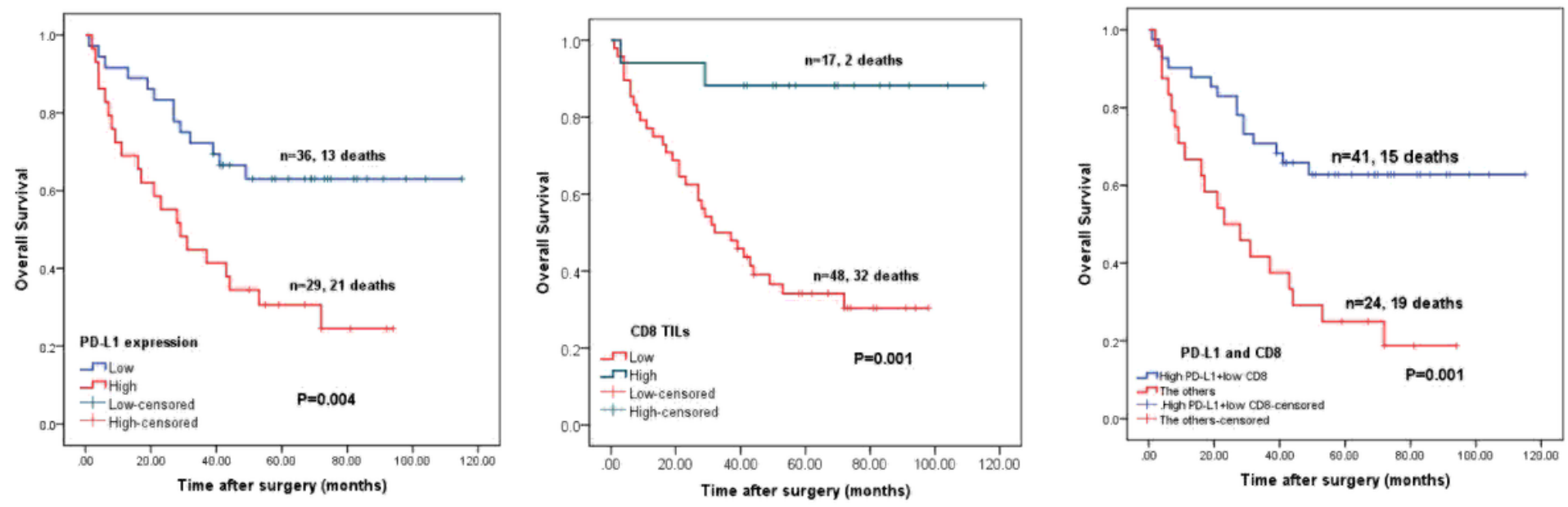

Figure 5

Correlation of PD-L1 and CD8 expression level and overall survival in the patients with cavitary LUAD. A: Patients with low PD-L1 expression showed better overall survival than that with High PD-L1 expression $(P<0.001)$. B: Patients with high CD8 TIL showed better overall survival than that with low CD8 TIL 
$(P=0.010)$. TIL: tumor infiltrating lymphocyte. C: Patients with high PD-L1 and low CD8 expression showed poorer overall survival than that with the other phenotypes $(P<0.001)$. 